\title{
Blood Lactate Response to Active Recovery in Athletes vs. Non-Athletes
}

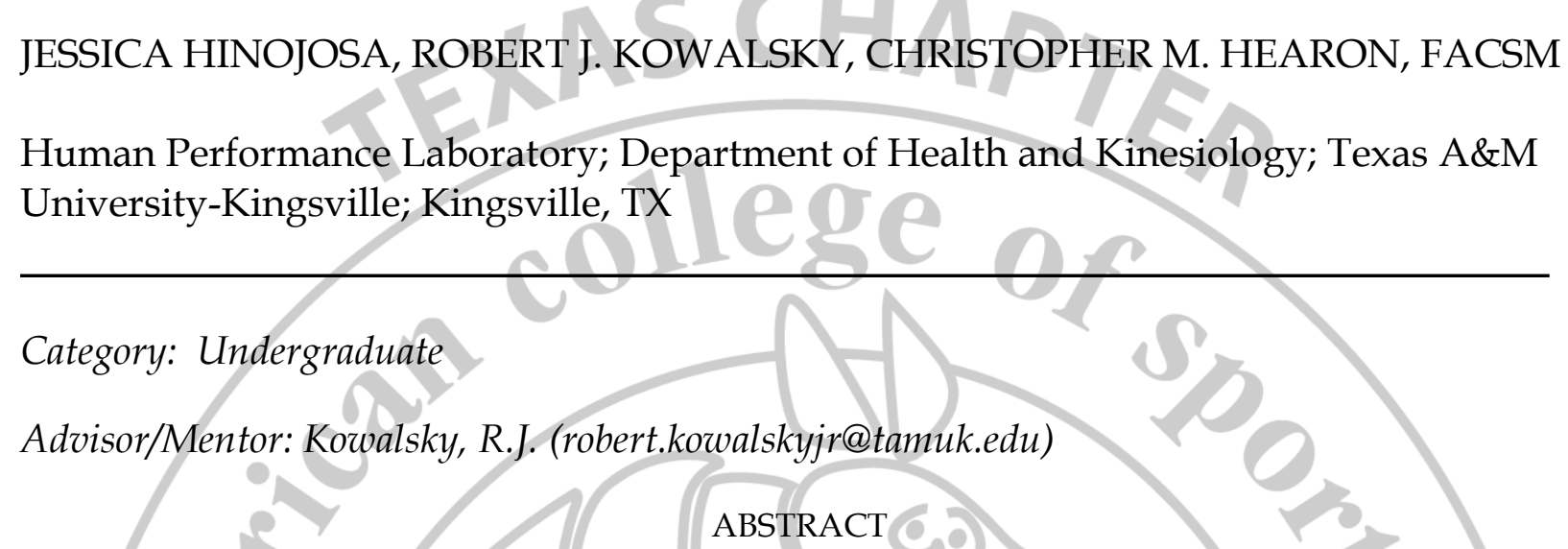

Previous research has indicated an optimal intensity for active recovery following a bout of exercise for the removal of blood lactate to be at $80 \%$ of the individual's lactate threshold. However, these previous research studies have relied primarily on highly trained athletes. Therefore, the $80 \%$ of lactate threshold might not be the optimal intensity for non-athlete populations attempting to recover from lactate producing exercise. Purpose: To investigate the acute blood lactate response during active recovery at $80 \%$ of lactate threshold for athletes $v s$. non-athletes following a vigorous bout of activity. Methods: Apparently healthy and active young adults $\left(N=16,56 \%\right.$ male, $21 \pm 1 \mathrm{yr}, \mathrm{BMI}: 26.5 \pm 3.6 \mathrm{~kg} / \mathrm{m}^{2}$, SBP: $122 \pm 13$ $\mathrm{mmHg}$, DBP: $71 \pm 8 \mathrm{mmHg}$ ) were enrolled in a cohort study design that separates subjects into highly trained collegiate athletes (participating in a NCAA sport) and non-athletes. Each subject had two study visits. Visit one included collection of subject characteristics including baseline values of blood pressure and heart rate via an automated device, and blood lactate via finger stick. Additionally, subjects underwent a Bruce Protocol on a treadmill to determine lactate threshold as well as $\mathrm{VO} 2_{\max }$. Visit two consisted of a treadmill warm up at $5 \mathrm{mph}$ and $0 \%$ incline followed by a brief running protocol at $90 \%$ of their $\mathrm{VO}_{2}$ max for 5 minutes. Immediately following the 5 minutes, subjects engaged in active recovery at $80 \%$ of the subject's lactate threshold until baseline lactate levels were reached or until 32 minutes of active recovery was completed. Measurements of heart rate and blood lactate were taken at rest, postwarm up, post-exercise bout, and every four minutes during active recovery for day two. Area under the curve (AUC) was calculated for blood lactate and general mixed linear modeling was used to compare AUC for athletes $v$ s. non-athletes while Cohen's $d$ was calculated to determine effect size. Post hoc analysis of individual time points used Bonferroni adjustment. Alpha level was set at $p<0.05$. Results: There was a significant difference in overall lactate $(\beta=-109.8 \mathrm{mmol} / \mathrm{L}, p<0.001, d=2.32)$ as well as at each time point during the active recovery (all $p<0.005$ ). There was also a significant difference in overall heart rate in favor of athletes having lower heart rate response throughout $(\beta=-14 \mathrm{bpm}, p=0.003, d=0.164)$. When individual time points were analyzed, there was a significant difference in heart rate at time point $7(\beta=-$ $20 \mathrm{bpm}, p<0.005, d=2.243)$, time point $8(\beta=-21 \mathrm{bpm}, p<0.005, d=2.305)$, and at time point $10(\beta=-18.533$ $\mathrm{bpm}, p<0.005, d=2.080$ ) in favor of lower heart rate for athletes. Conclusion: Active Recovery at $80 \%$ of lactate threshold demonstrated a significant difference in the removal of lactate for athletes compared to non-athletes. Future research should investigate the most efficient workload for the removal of blood lactate in non-athletes, potentially an intensity that lowers non-athletes heart rates more than the intensity used in this study. 